\title{
A REVIEW OF MODEL BASED AND DATA DRIVEN METHODS TARGETING HARDWARE SYSTEMS DIAGNOSTICS
}

\author{
Christos SKLIROS, Manuel ESPERON MIGUEZ, Ali FAKHRE, Ian K. JENNIONS \\ IVHM Centre, Cranfield University, College Road, Bedfordshire, MK43 0AL, UK, \\ C.Skliros@cranfield.ac.uk
}

\begin{abstract}
System health diagnosis serves as an underpinning enabler for enhanced safety and optimized maintenance tasks in complex assets. In the past four decades, a wide-range of diagnostic methods have been proposed, focusing either on system or component level. Currently, one of the most quickly emerging concepts within the diagnostic community is system level diagnostics. This approach targets in accurately detecting faults and suggesting to the maintainers a component to be replaced in order to restore the system to a healthy state. System level diagnostics is of great value to complex systems whose downtime due to faults is expensive. This paper aims to provide a comprehensive review of the most recent diagnostics approaches applied to hardware systems. The main objective of this paper is to introduce the concept of system level diagnostics and review and evaluate the collated approaches. In order to achieve this, a comprehensive review of the most recent diagnostic methods implemented for hardware systems or components is conducted, highlighting merits and shortfalls.
\end{abstract}

Keywords: diagnostics, system diagnostics, condition based maintenance, model based diagnostics, data driven.

\section{INTRODUCTION}

Integrated Vehicle Health Management (IVHM) is a capability that enables condition monitoring of high value industrial assets and offers numerous services to stakeholders [1]. IVHM outputs can be applied to optimize individual project plans as well as an organization's overall business plan. The understanding of a system's fault modes can provide feedback for the design of new products that can then be made to be more robust to faults and embed intelligent fault detection features.

Maintenance costs contribute significantly to the overall operational cost of high value industrial assets [2, 3]. Thus, optimization of maintenance activities can substantially contribute to overall operational cost reduction. One of the most important services that IVHM can provide is a provision of Condition Based Maintenance (CBM) [4]. CBM targets in suggesting maintenance actions before the performance of an asset has deteriorated to a level that will lead to an unscheduled interruption of its operation [5]. This leads to improved and more robust maintenance plans and potential cost reduction strategies. CBM is based on the implementation of intelligent diagnostic and prognostic methods. Diagnostics is a process of accurately detecting system faults and isolating their root cause. Prognostics moves a step further and targets in identifying the Remaining Useful Life of a system or a component before its replacement. There has been a profound interest from both Original Equipment Manufacturers
(OEMs) and operators to advance their level of scientific understanding in the subject area in order to provision optimized maintenance scheduling of high value assets as well as to better align their business models to market needs [1].

The main contribution of this paper is a review and discussion of system level diagnostics. A literature review of publications developed under this approach is conducted. A discussion is offered that identifies the main features of this approach, merits it can provide, limitations it faces and areas of further research.

In order to set the framework for system diagnostics, a review of existing diagnostic methods is presented. An algorithm-based taxonomy of existing methods is proposed. A literature review of the most recent and representative techniques of its respective category is conducted. Advantages and disadvantages of each category are outlined and the way these techniques were applied to system level diagnostics is discussed.

\subsection{Scope of present work}

System level diagnostics approach is especially beneficial in applications of high value industrial assets whose downtime for unscheduled maintenance is expensive. This approach suggests quickly and accurately to a system's maintainer the component that should be replaced to restore the asset to operational condition.

A definition of "system" in engineering terms is quite generic. In this paper, the concept of a system 
will be limited to a hardware system which consists of components that can be physically replaced. Approaches that consider a computer software as a system and the lines of the code as its components will not be considered [6].

Diagnostic methods developed so far focus on the component level and, sometimes, on the system level. In order to clarify this distinction, an example of a compressor (component) in a gas turbine engine (system) is considered. Applying a diagnostic analysis on the engine (system level), the major interest is detecting a fault in the system and identifying the component (e.g. compressor) that if replaced will restore the system to the healthy state. Under a system level diagnostic analysis, the root cause of the component's fault is not the main concern. In the example of the compressor fault, the root cause could be related to fouling, tip clearance, erosion, corrosion, foreign object damage [7].

In the light of the aforementioned background, the main objective of this paper is to review and discuss the work conducted on system level diagnostics. The main features of this approach are introduced, methods that were developed under this approach are presented along with their advantages and limitations.

Discussion of system level diagnostics, prerequisites a review and discussion of the main features, advantages, and shortfalls of existing diagnostic methods. The quest for each stakeholder is to employ the most suitable diagnostic method(s) that will enable the optimum utilization of the data acquired from their asset of interest to optimize maintenance. Therefore, knowledge of the key pros and cons of each available method plays a vital role on provision the implementation of the most suitable diagnostic method. Thus, another contribution of this paper is a review and discussion of the most recent public domain literature dedicated towards the diagnostic problems. A taxonomy that categorizes diagnostic methodologies based on the algorithms they use is proposed. Initially, diagnostic reasoning techniques that can be used individually or in combination with other diagnostic methodologies are presented. This paper details model based and data driven methods. Methodologies based on expert systems are outlined since they offer a unique approach, but the discussion conducted is limited. Finally, applications of combining model based and data driven methods and creating hybrid techniques are described.

It is to be noted that the aim of this work is not to conduct an exhaustive search of publications of each category but to discuss the most recent work conducted under each respective approach. This work refers to methods that have been developed in order to apply diagnostics in a systematic way. However, it should be mentioned that since diagnostics is an engineering problem, heuristic methods can always be part of the solution (e.g. cracks or leaks at mechanical systems can be discovered with visual inspections or nondestructive inspections [8]).

The remaining part of this paper is organized as follows. In section 2, a taxonomy of diagnostic methods is proposed and fault reasoning techniques are presented. In section 3, rule and case based reasoning methods are presented. In section 4, model based diagnostic techniques are illustrated, while in section 5 data driven techniques are described. In section 6, hybrid techniques that combine model based and data driven methods are outlined. In Section 7, the system level diagnostic concept is presented and a literature review of the most important publications is conducted. In Section 8 and 9, a detailed discussion and concluding remarks are elaborated respectively.

\section{TAXONOMY OF DIAGNOSTIC METHODS AND DIAGNOSTIC REASONING TECHNIQUES}

Categorization of diagnostic methods can be conducted in many ways depending on predefined criteria. For example, a taxonomy of diagnostic algorithms can target to categorize methods based on their application to various fault severity modes (e.g. minor degradation, major degradation, complete destruction). Another categorization of diagnostic methods can separate them in qualitative methods that conduct the analysis using qualitative criteria of system parameters (e.g. increase in a temperature, decrease in mass flow), or in quantitative methods that extract their results by comparing system parameters on predefined thresholds (e.g. component electrical resistant higher that $10 \mathrm{k} \Omega$ ). In this paper, a taxonomy of diagnostic methods is proposed using an algorithmbased perspective. Figure 1 presents a taxonomy of the compiled diagnostic methods. Under this approach diagnostic methods are categorized based on the features of the algorithms they are using, regardless of qualitative or quantitative characteristics. Existing diagnostic methods in the literature can be characterized into three main categories:

1. Model Based Methods (Physics based)

2. Data Driven Methods (Artificial intelligence, Statistics)

3. Expert System Methods (Rule base or Case base reasoning)

A discussion that is conducted for each respective category informs the reader for the inputs each one requires and compares their advantages and disadvantages. Also, the analysis of each respective category compares the advantages and shortfalls of various algorithms in each category (e.g. accuracy, response time, ease of application).

At this section, techniques that can be used in combination with the diagnostic methods and will be referred in this paper as "Diagnostic Reasoning 


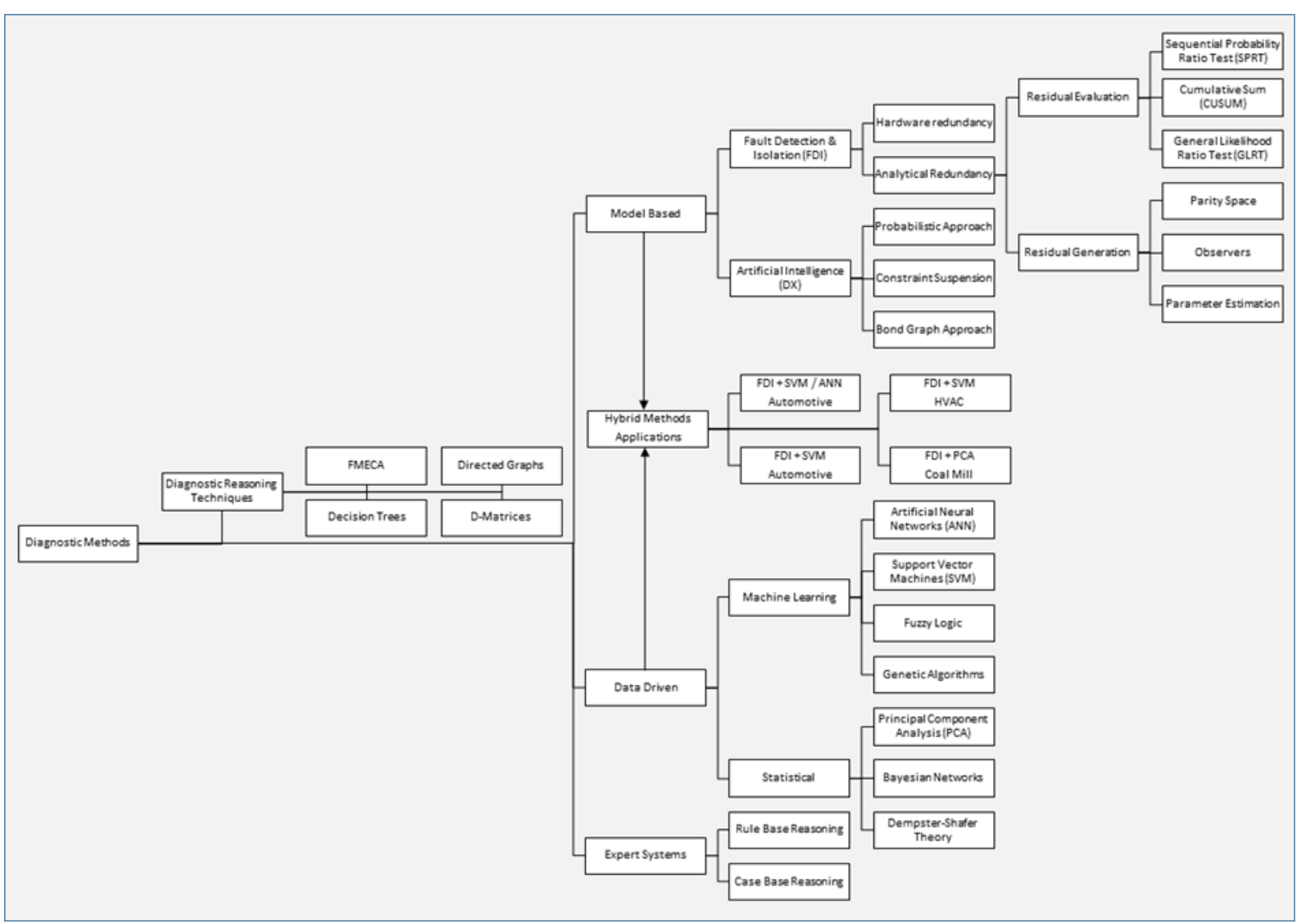

Fig. 1. Taxonomy of Diagnostic Methods \& Diagnostic Reasoning Techniques

Techniques (DRT)" will be presented. DRT can either be used individually when conducting a diagnostic analysis or can be used as a preprocessor in order to enhance the accuracy and response time of the results. The most widely used DRT are Failure Mode Effect and Criticality Analysis (FMECA), Decision (Fault) Trees, Directed Graphs and Decision Matrices (DMatrices).

A FMECA breaks down the system into its subsystems and components and works in a bottomup way starting from the failure modes each component can present and propagates its effects upwards to the higher system levels. As it is described by [9], a system's FMECA analysis answers the questions: "what problems could arise?", "how likely are these problems to occur and how serious are they if they happen?", "how can these problems be prevented?". In [10] there is conducted a FMECA study on an industrial gas turbine. The analysis conducted had two branches. Two separate FMECA are conducted on the gas generator subsystem and on the lubrication subsystem. The FMECA was based on onedimensional models of the respective subsystems. The results of this study could identify failure modes in components in each respective subsystem, based on their effect on the overall system performance. Another application of FMECA, as suggested in [11], is that it can provide a database of system fault modes, which a diagnostic method should target to identify.

A Decision Tree models the system in the same way as a FMECA (higher levels-major subsystems, lower levels-components). However, the diagnostic analysis is conducted by traversing the tree topdown. Higher level nodes are related to major subsystems and lower level nodes correspond to components or different failure modes in the same component. At every node of the tree, a system's parameter is compared to a baseline value and depending on the result of the comparison lower branches of the tree are being excluded. The algorithm terminates when it reaches the lowest nodes of the tree. Depending on the fault modes that the analysis targets to capture, different architectures can be developed. For example, in a pipe fault case, the decision tree should be constructed differently depending if the analysis just targets to capture the faulty pipe or it targets to capture the root cause of the pipe failure (e.g. leakage, blockage). In [12, 13], was developed a fault tree of an aircraft fuel system. Fuel tank imbalance faults were considered at higher levels of the trees and faults such as pump failure or pipe leakage were modelled on the lower levels. The proposed methodologies could capture the injected faults. Ambiguity groups among some components were developed in few cases.

Another way of identifying possible system and component fault modes is the Directed Graph 
analysis. The nodes of the directed graph represent the system components failure modes and are connected with directed and weighted edges. The edges indicate the effect that a component fault has on other components that are connected with. Starting from a node where the observed values deviate from normal, the algorithm back-traces the directed graph and all possible fault combinations can be identified. The authors in [12] conducted a diagnostic analysis on an aircraft fuel system, using a directed graph. In this case study, a blocked valve node is connected to a fuel flow node with a weight of "-10", while nodes of pipes that have normal fuel flow operation are connected with a weight of " +1 ".

Considering a system with its corresponding sensors, a Decision Matrix (D-Matrix) is a table with its columns and rows corresponding to the system failure modes and sensors respectively. The values of the matrix can be either binary or real numbers and represent the way each failure mode is reflected to each sensor. In [14] there are reviewed different techniques for creating and integrating Dmatrices. The D-matrices are separated into three categories; Engineering D-matrix (ED), which is extracted from a physics based analysis of the system; Document D-matrix (DD), which is extracted from maintenance logs and Historic Dmatrix, which can be built utilizing historic knowledge that correlates various symptoms to their corresponding failure modes. Integration of diverse types of D-matrices requires expert or domain knowledge of the application under examination. By using a D-Matrix, the designer understands the critical measurements that reflect each component's health state. This can result in the development of more accurate and faster diagnostic rules.

\section{EXPERT SYSTEM}

Expert system methods contain the rule based reasoning approach and the case based reasoning approach. Rule based reasoning is a technique in which the diagnostic results are extracted by propagating the system's observations through a set of rules. The embedded rules are derived from the knowledge of an expert or a group of experts. An expert system can be defined as: "A computer program designed to model the problem solving ability of a human expert" [15]. There are two ways of building an expert system, forward chaining reasoning and backward chaining reasoning. The forward chaining technique propagates the system observations through the expert system's rules and has diagnostic results as its output. In the backward chaining, a fault hypothesis is initially suspected and the expert system investigates if the hypothesis can be supported from the observations, using a predefined set of rules.

Case base reasoning is a methodology of solving an existing problem based on the knowledge acquired by solving the same or similar problems in the past. Under the case base reasoning approach, old solutions and outputs of old problems that are similar to the case under examination are collected. Selecting the old case that most resembles the case under examination, based on predefined criteria, an initial solution is proposed. Through a series of steps involving adjustment and modification of the initial solution, the final solution is extracted. In the case of diagnostics, the solution may have the form of a specific failure mode on a system.

The methods of rule and case based reasoning require the system expert knowledge of experienced personnel or a large historic database of solutions of various cases. Both prerequisites are hard to acquire in many cases. When a diagnostic analysis is conducted based on limited experience of the system failure modes, the corresponding symptoms or successful troubleshooting actions, the rule and case base reasoning methods are not applicable. This paper focuses on reviewing the developed diagnostic methods in applications where little or no historic knowledge of the system failures and troubleshooting is available. To this end, the next sections discuss model based and data driven methods in more detail.

\section{MODEL BASED METHODS}

Model based methods, use a physics model of the system or component under examination, to conduct the analysis. Physical parameters calculated by the model are compared with system observations and by using various techniques faults can be detected and their root cause can be isolated. Model based methods can be further separated into two major categories, an approach developed by the Control Engineering community, Fault Detection and Isolation (FDI), and an approach developed by the Diagnostic Artificial Intelligence (DX) community.

\subsection{Fault Detection and Isolation (FDI)}

Considering a physical system the FDI approach considers two alternative methods, hardware redundancy and analytical redundancy. The methodology used by both techniques is based on creating redundant information for a system under examination. By analysing the residual vector created by comparing the redundant system information to the observed measurements faults can be detected. In hardware redundancy, redundant information is generated from a hardware module (component or sensor) that operates in parallel with the main hardware module. In analytical redundancy, a system simulation model generates the redundant information.

\subsubsection{Hardware Redundancy}

Hardware redundancy is a method that has been applied mostly in the designing of fault tolerant systems. The main target of hardware redundancy is 
to guarantee the safety of the system when a component or sensor fails. This is accomplished by installing redundant hardware (components or sensors) operating in parallel. A major disadvantage of hardware redundancy is that increases in system size, weight, power consumption and cost [16].

Hardware redundancy has fault detection capabilities as well [17]. This is accomplished by considering two system modules (components or sensors) that work in parallel and a comparator box that compares the modules values. A fault can be detected when an inconsistency is observed. This technique cannot isolate the faulty module, but it can detect a fault in one of the two compared modules [16].

\subsubsection{Analytical Redundancy}

Analytical redundancy methods conduct an analysis on the residual between a system's observations to its simulation model. An analysis is conducted on the generated residual in order to detect faults in the system (residual evaluation). Analytical redundancy algorithms target in creating residuals insensitive to sensor and process noise but sensitive to system faults (residual generation). The generated residual must be close to zero at a system's healthy state and greater than zero when faults exist. The main advantage of analytical redundancy compared to hardware redundancy is that this technique does not require additional hardware. On the other hand, a limitation of this method is that diagnostic accuracy is highly dependent on the fidelity of the simulation model.

Residual evaluation targets in decreasing the false alarm rates and building algorithms robust to noise. Statistical hypothesis testing techniques are used for residual evaluation. The null hypothesis is defined as the fault free case. Observing the system parameters, if the null hypothesis is considered to hold, no faults are detected. When the null hypothesis is no longer valid, a fault is detected [18]. Rejecting or confirming the null hypothesis is based on rules derived from probability theory. For example, if a $95 \%$ confidence rule is set, having a dataset of pressure measurements against time and $95 \%$ of the measurements are within a predefined healthy threshold the system can be considered healthy, otherwise, a fault is detected. The most popular hypothesis testing algorithms used in diagnostics are: Sequential Probability Ratio Test (SPRT)[19], Cumulative Sum (CUSUM) algorithm [20] and General Likelihood Ratio Test (GLRT) [18]. Hypothesis testing algorithms can be designed to detect either single faults or multiple faults [21].

Residual (parity) space method is a residual generation technique. Under this approach, system inputs and outputs are represented by vectors. Using a simulation model that is built on mathematical equations, output system values are predicted. Thus, the residual is the algebraic difference of the observed and the simulated output vector. The simulated output vector depends on constants defined in the system's mathematical equations. These constants are selected in such a way that produce a residual close to zero at a fault free case and residuals higher than zero when faults exist [22]. A fault diagnostic analysis based on parity space method was conducted in [23] on a brushless DC motor. The proposed methodology considered the mechanical and the electrical system of the motor operating in isolation (no interconnection between them) and could accurately detected injected faults in each one of them.

An alternative technique for residual generation is based on optimal estimation algorithms (observers). The difference this technique has compared to parity space method is that a system's simulation model is not based on mathematical equations but on optimal estimation algorithms (Kalman filter, weighted least square, etc.) that can estimate a system's outputs based on its inputs [24]. Under this approach, specific faults can be isolated selected by the algorithm's designer. This can be done by considering all other faults as noise and selecting the constants of the simulation model appropriately in order to produce residual higher than zero only in the presence of a predefined fault [25-29].

Residual generation can also be conducted using a technique called parameter estimation [30, 31]. Under this technique, a system is modelled using mathematical equations and deviation of its nominal operation (healthy state) can be modelled by adjusting properly equations parameters. These parameters represent features of the physical components, e.g. resistance for electrical components, or effectiveness for mechanical components. Based on the observed input and output measurements, the physical parameters can be estimated using optimal estimation techniques (Kalman filter [32, 33], weighted least squares [34]). Faults are detected by correlating parameters that deviated from their nominal baseline to system components. For example, a decrease in the value of a parameter representing a heat exchanger's efficiency in an air conditioning system suggests degradation in the heat exchanger component. A parameter estimation method has been applied on a brushless D.C. motor considering separately the mechanical subsystem and the electrical subsystem of the motor [35]. Two faults were injected into each respective subsystem and the proposed methodology could capture the deviations on the physical parameter values and attribute the faults to the respective components. Parameter estimation, has been widely applied in gas turbine diagnostics (Gas Path Analysis - GPA), initially by [36] and further developed by [34, 36-41].

\subsection{Diagnostic Artificial Intelligence (DX)}

A model based approach to detect and isolate systems faults was developed by the Diagnostic Artificial Intelligence (DX) community [42]. This 
approach models a system as a network of interconnected components and each component is modelled by physical equations (constraints) that describe its nominal operation. Providing input values to the model, physical parameters can be calculated at the input and output of all components. A fault is detected when estimated physical parameter values (model) deviate from observed physical parameter values (sensors) [43].

\subsubsection{Fault detection challenges}

Fault detection is highly dependent on the model's accuracy, in [43] there is developed a method of adjusting properly the component equations to be insensitive to noise and disturbances, but sensitive to faults. Based on the complexity of the modelled system and the model's capability of incorporating noise and disturbances, there have been proposed diagnostic rules based on fixed thresholds [44] or adaptive thresholds [4547].

A major obstacle observed in general in all fault diagnostic applications is a trade-off between probabilities of false alarms to probabilities of misdetections. Under DX approach, in [46] there is developed a technique that was able to quantify these probabilities and select a fault detection threshold that could minimize them. Another method of controlling false alarms to misdetections ratio was proposed by [48]. For every observed parameter, a rule is defined that the fault threshold should be exceeded at least " $x$ " times at " $y$ " consecutive time slices, in order for a fault to be detected.

\subsubsection{Fault isolation challenges}

At the fault isolation step, based on the functional interconnections among the components, all possible combinations of components that can theoretically be faulty are identified. The number of the identified sets grows exponentially in the number of components. In cases of complex systems with many components, the exhaustive search algorithm for identifying possibly faulty components, leads to algorithms with high computational time $[49,50]$. Stochastic algorithms [51] and machine learning algorithms [52] have been used to solve the problem more efficiently.

One of the main techniques used for fault isolation is the probabilistic reasoning approach. Under this technique, a probability is assigned to each set of possibly faulty components, based on the existing observations (available sensor measurements). The assigned probabilities are based on Bayes rule and are updated every time a new sensor observation becomes available. A-priori probabilities used Bayes rules are usually calculated from statistical data describing component faults [49]. The authors in [50] proposed another methodology of assigning probabilities to components being faulty. At this methodology, each component is assigned a probability of being faulty, not based on the available sensor measurements, but based on the number of possibly faulty sets it is contained in. It was proved that the latter approach can produce accurate fault isolation results without considering all possible fault combinations of faulty components, thus the computational time of the method was reduced.

Constraint suspension introduced by [42] is an alternative fault isolation technique. Under this approach, components are serially excluded from the system model and physical parameter values are not propagated through their equations. Every time a component is excluded, values of system's physical parameters are propagated through the remaining model components. If inconsistencies continue to be present between the estimated and the observed values, then the suspended component is not considered to be faulty. However, if after excluding a component, estimated and observed values are consistent, this component is suspected to be faulty.

A limitation this technique faces is that there may be cases where the suspension of a component may result in insufficient number of parameter values to conduct consistency check for all remaining components [44, 48]. In such cases, ambiguity groups are created. A component "comp_l" belongs to the ambiguity group of component "comp_2" if by excluding component "comp_2" from the model, consistency check cannot be conducted to the input and output values of component "comp_l”". Formation of ambiguity groups depends on the existing sensor suite, the equations used for modelling components and the connection types [53]. A case study on the NASA rover's power system was conducted in [53] comparing the ambiguity groups formatted depending on the different sensor suites installed. The selection of the appropriate sensor suite depends on the specific application in order for the algorithm to be able to identify faults and usually it targets the most critical components.

\subsubsection{Fault detection challenges}

A fault diagnostic approach has been developed based on system modelling using bond graphs [5457]. Using a bond graph model a cause-effect relation can be developed among a system's physical parameters. For example, fluid pressure (cause) in a tank can generate fluid flow (effect) in a pipe. When faults occur, positive or negative deviations (inconsistencies) are captured between the observed and the modelled values of the system's causes or effects. Based on the deviation of its nominal value (increase or decrease) causeeffect relationships can detect possible qualitative deviations of component characteristics (decrease in the effectiveness of mechanical components). In most applications, there exist more than one faulty candidate components. In such cases, each candidate fault generates a different rate of change 
of the model's physical values (causes and effects). Comparing each fault's cause-effect deviation signature, with measured system's values the faulty component can be isolated.

\section{DATA DRIVEN METHODS}

Diagnostic analysis conducted by data driven methods is based on machine learning algorithms and statistical algorithms. Data driven algorithms correlate system observed measurements with a health state (healthy or faulty) and solve regression and classification problems (machine learning) or assign a probability of a system or a component being healthy or faulty (statistical). These techniques have been proved to be successful in isolating both component and sensor faults.

It is noted that the methods that will be presented at the next sections are the most popular techniques applied for diagnostics. There exist many different variations of data driven algorithms. For example, there have been developed different types of Artificial Neural Networks (Probabilistic, Dynamic, etc.) or different dimensionality reduction algorithms (Principal Component Analysis, Non-Linear Principal Component Analysis, Partial Least Squares, etc.) that are not explicitly discussed in this paper. However, their fundamental characteristics and their main objectives fall into the methods described in the next sections.

\subsection{Machine Learning (ML)}

Machine learning is a field of computer science also called soft computing. Machine learning targets in estimating the output of a mathematical problem for any given input, by developing a set of rules derived from discrete solutions of the problem under examination. There have been developed various algorithms that propose different ways for developing the estimation rules (Artificial Neural Networks, Support Vector Machines, etc.). Machine learning is currently one of the most rapidly developing field in computer science with application on fields such as; image recognition [58] and economics [59], since it has been proved to provide accurate solutions in complex problems that analytical methods fail. At this section the most popular techniques applied on diagnostic problems will be discussed.

\subsubsection{Artificial Neural Networks (ANN)}

The basic principle of neural networks is the mapping of a set of inputs to a set of outputs under the influence of weights and thresholds. Every input vector corresponds to a desired output vector and the goal is to adjust the weights and the thresholds of the neural network to create an output vector as close as possible to the desired vector. The appropriate adjustments of the neural network weights and thresholds permit an optimal mapping (empirical risk minimization) [60]. To assign the appropriate weights and thresholds of the neural network an optimization problem must be solved (training of the network) and the computational time for training a neural network is linear in terms of the depth of the network [61].

The underlying dangers of using ANN are: i) divergence of the algorithm, ii) trapping in local extrema and iii) overfitting [62]. Divergence and trapping of the algorithm on local extrema is a consequence of using deterministic optimization algorithms in the training stage. Techniques that can mitigate the risk of divergence and trapping of the algorithm in local extrema due to deterministic optimization algorithms can be found in [63]. Overfitting of a neural network is the situation where the network over-conforms to the training examples and cannot produce reliable outputs for new examples [62]. A major reason for the overfitting of a neural network is the use of too many training examples. A rule of thumb to avoid overfitting, suggested in [62] is that the number of trainable weights must be smaller than the number of samples. ANN can model physical systems with respect to non-linearity [62] and have been used in diagnostic applications.

Component fault detection and isolation is approached using ANN by two different techniques. Under the first technique, a neural network is trained in such a way that using the operating conditions as inputs, produces the estimated system's values. The estimated values are compared with the real system measurements and when appropriate thresholds are exceeded faults are detected [64-66] Under the second technique, a neural network gets as inputs the system measurements and estimates the system physical parameters. This approach can detect a fault and link the abnormal physical parameter to its corresponding component [67-69].

An architecture of ANN called Auto Associative Neural Networks (AANN) that map an input vector to itself was proposed by (70). In cases where the input vector represents sensor measurements, AANN have been proved successful in noise filtering. Applications of pre-processing sensor measurements for noise filtering on gas turbines using AANN have been discussed in [7174].

\subsubsection{Support Vector Machines (SVMs)}

Support vector machines were initially introduced in [75]. The target of SVM is to separate data points with distinct characteristics in an optimal way. An optimization problem is solved using Laplace multipliers and KKT (Karush-KuhnTucker) conditions. SVM selects a linear function to separate the data points in two-dimension space. If the data cannot be linearly separated into two dimensions, they are projected into higher dimensions using kernel functions (polynomial, 
radial-basis, sigmoidal) where can be linearly separated. A shortfall of SVM classification is their slow response and as a mitigation action, optimization techniques that can select the appropriate parameters used into the method's mathematical equation have been proposed in [76].

SVM have been used widely in gas turbine diagnostic problems by utilizing their optimal classifier feature. SVM can be trained on a dataset of engine measurements, containing both healthy and faulty engine states. Therefore, when engine measurements enter a SVM, they can be classified as either healthy or faulty [77] or linked to specific faults [78-79].

A technique that combined ANN and SVM applied on a vehicle, was proposed by [80]. At the first stage, an ANN is used to detect faults by comparing the residual between the input and the output to a nominal threshold. The fault isolation is conducted by a multiclass SVM.

\subsubsection{Fuzzy Logic (FL)}

Fuzzy logic is a mathematical technique derived from set theory that has been applied to fault diagnostic problems. The authors in [81] used fuzzy logic both for fault detection and for threshold adaptation. Initially, a residual was created between a system's observed values and its mathematical model. Fault detection using fuzzy logic is conducted in three stages: fuzzification, inference and defuzzification. During the fuzzification step, membership functions for various residual groups are defined. Each data point of the residual space is assigned a degree of membership at each residual group. The next step is the inference, where each fault is corresponded with one or more residual groups. Finally, in the defuzzification step, each fault case is associated with a crisp value under predefined rules [82].

The most current trend is the combination of fuzzy logic with classification algorithms (ANN, SVM). Fuzzy logic is used in the initial stage in order to cluster the observed parameter into fuzzy sets (fault modes) and a classification algorithm is trained to classify system parameters to its corresponding health state. In [83] there is conducted a diagnostic analysis on a Heat Ventilation and Air Condition system (HVAC) using fuzzy logic and neural networks. At the fuzzy logic stage, degrees of membership to five fuzzy sets (healthy, temperature sensor error, valve position sensor error, valve stuck open, valve stuck closed) were assigned at three system parameters (temperature, valve position, air flow). A neural network was trained to classify the system parameters to its corresponding set. The proposed methodology was applied to simulated and experimental data and could accurately detect and isolate the system's faults. In [84] there is developed a fault diagnosis algorithm for a wind turbine. The proposed algorithm uses a kernel fuzzy c-means clustering algorithm to cluster vibration signals into fuzzy sets (fault modes). The fuzzy sets are used for training a multiclass SVM that classifies input parameters to their corresponding fault modes. The proposed methodology was compared to a multiclass classic SVM and an ANN, without using the fuzzy logic stage, and demonstrated higher accuracy. The authors in [85] use a fuzzy c-means clustering algorithm to cluster exhaust gas temperature data from an industrial gas turbine. The fuzzy dataset is used to train and test a multiclass SVM on the failure modes that had been identified in the clustering step. A neural network was also tested for the data classification but the SVM demonstrated higher accuracy.

\subsubsection{Genetic Algorithms (GA)}

Genetic algorithms are stochastic optimization algorithms. The advantage they have compared to deterministic optimization methods is that they do not require the computation of the gradient of a function, so the objective function is not necessary to be differentiable. Their disadvantage is that the computational time required is much longer compared to the deterministic methods [86].

Genetic algorithms have been utilized in gas turbine diagnostics, both for sensor diagnosis [87, 88] and for components diagnosis [87, 89-91]. An objective function that incorporates the residual between a system's simulated measurements and the real observations is defined. The variables of the objective function are the components physical parameters, which will be estimated using GA. Observing the residual between a parameter's estimated values compared to its nominal values, faults can be detected at the corresponding components. In gas turbine applications genetic algorithms demonstrated better diagnostic results when compared to the Gas Path Analysis (GPA) method [89. 91], as well as with neural networks and fuzzy logic [89].

A serious drawback of using GA is their slow response. Techniques have been proposed to decrease the high computational time of GA. In [87] there is proposed a technique in which only specific parameters of the objective function are allowed to deviate. These parameters are selected based on engineering judgment of each specific application. In [90] there is proposed a strategy that allows the algorithm to make bigger steps at the initial stages, so the search is accelerated. The authors in [89] introduce the response surface method, which allows the algorithm to discard members of the population that are not in the region of the global minimum at the initial stages.

\subsection{Statistical Methods}

Techniques derived from statistics and probability theory can be used to conduct diagnostic analysis. Statistical methods can be used to detect outliers in datasets which can either be a 
pre-processing part of the analysis or can suggest faulty cases. Principal Component Analysis (PCA) has been mostly used for this kind of applications. Also, Bayesian probability analysis and DempsterShafer theory can offer results presenting the probability of occurrence of a fault mode based on the measured parameters.

\subsubsection{Principal Component Analysis (PCA)}

PCA is a statistical technique that maps high dimension datasets into lower dimensional space. The lower dimensional data are called principal components of the initial dataset and are selected to maintain the variability existing in the original data [92]. In fault diagnostic applications, especially in cases where the data size is large and many data point have almost identical variances, it is beneficial to conduct a PCA on the initial data set in order to reduce the size of the data to be processed.

PCA technique for fault diagnosis can either be used as a pre-processor so that reduced dimension data can be analysed from another algorithm. In [64] PCA was applied on data from a rotating machinery and the principal components were used to train a neural net. In [93] there is conducted a diagnostic analysis on an electronic device that is used to monitor human pulmonary functions. Initially, PCA is conducted on healthy data. A kmeans clustering algorithm is applied on the reduced dimension principal components to identify the various patterns existing for the various operational modes. The $\mathrm{Q}$ test $(\mathrm{Q}$ test is a mathematical technique used to identify outliers out of a sample of measurements) was used to define the appropriate threshold for each cluster. The fault isolation step is conducted using a neural network. When a fault is detected, a neural network, that has been trained to predict sensor values, is used. Data collected a few sampling points before the fault occurrence are introduced to the neural network and if the predicted values are different from the observed values, the sensor is declared faulty. The proposed methodology was applied on real system's data and could accurately identify the injected sensor fault.

The authors in [94] introduced a technique called Multi Scale Principal Component Analysis (MSPCA). The proposed methodology, applied at a chemical plant, leverages both the advantage of the wavelet transform that considers the correlations within sensors and PCA that captures the correlations among the sensor measurements. The system data are decomposed on various levels from the wavelet transform and at the next step are analysed using PCA. Using the Q test appropriate thresholds for fault detection can be established. The proposed methodology was tested on real system data and demonstrated better diagnostic results compared to the conventional PCA without pre-processing the data using the wavelet transformation. The authors in [95] developed a diagnostic methodology that incorporates wavelet transformation and PCA for a building air handling unit. The data are pre-processed by the wavelet transformation and the low frequency features are excluded from the analysis because they are due to weather or load variations and not due to component faults. PCA was applied on data from the real system in the fault free case before and after the wavelet pre-processing. The results demonstrated that when the data are not preprocessed false alarms are flagged. The corresponding thresholds were defined using Q test. The proposed methodology was tested on faulty cases and could accurately identify the existing faults.

\subsubsection{Bayesian network}

Bayesian network architecture is a way to represent the joint probability of various events by means of capturing the effects each one of them has upon the others. The three main characteristics of a Bayesian network are: i) that each problem variable is represented by a single node, ii) the nodes are connected by directed edges formatting a directed acyclic graph, and iii) each node is assigned a conditional probability distribution (with the parent nodes as parameters). An important prerequisite of constructing a diagnostic Bayesian network is the estimation of the a priori probability of the system's variables that are used in Bayes rule [96]. The determination of the a-priori probability can be based either on historical data or on expert knowledge. Bayesian networks have been used successfully on diagnostic applications, since they have the advantage of using probability assignment on the fault detection and isolation task that are useful in the design procedure of the diagnostic algorithms as well as in decision making [97].

In [98] a Bayesian network methodology for fault diagnosis is proposed. The architecture of the network considers three levels (causes, symptoms, faults). The faults are categorized in catastrophic or degraded. Depending on the way the causes, symptoms and faults are interconnected, they are categorized in multiple or common causes/symptoms and cascading faults. Appropriate probability thresholds are assigned for the algorithm to classify each case. Using the node interconnections, sensitivity analysis is conducted to investigate which of the causes or symptoms contributes more to the faults. The proposed methodology was applied on a power plant's centrifugal compressor and the results demonstrated that the proposed metrics could accurately capture the causes or symptoms that affected the faults under investigation.

In $[99,100]$ diagnostic Bayesian networks were used on a building's AHU. There are three kinds of nodes in the network (evidence nodes, fault nodes, additional information nodes). The evidence nodes observe sensor readings or a component's mode of 
operation and direct to fault nodes. The additional information nodes capture data derived from visual inspections, historic logs and other heuristic sources of information and enhance the diagnostic accuracy of the algorithm or point out meaningful inspections to the technical personnel. The proposed method was tested on experimental data and could successfully detect and isolate component faults. The advantage of this method is that it was developed without considering fault data, thus, it can be implemented in cases were historic fault data are unavailable.

\subsubsection{Dempster-Shafer theory}

Another technique derived from the probability theory and has been applied to fault diagnosis is Dempster-Shafer (D-S) theory. Under the D-S framework, degrees of belief are assigned to individual members or subsets of a set of events based on the existing evidence. The main difference compared to Bayesian network approach is that the belief value of a fact and its corresponding negation are not necessarily complementary [101].

In [102] there is developed a diagnostic methodology using D-S theory, that was applied to an aircraft's Auxiliary Power Unit (APU). The aim of the proposed methodology was to calculate the probability of multiple faults existing in the system. Different diagnostic algorithms are used to detect faults in the various subsystems existing in the APU (i.e. gas generator subsystem, fuel subsystem, etc.). Calculation of belief values for each algorithm under the D-S framework are based on various evidence (sensors, features and method each algorithm uses). Diagnostic algorithms are classified as highly dependent, weakly dependent or independent based on the overlap of the evidence they use. Using D-S fusion rule on the results of highly dependent, weakly dependent and independent algorithms, belief values of the existence of various fault modes are created. The step forward of the proposed approach is that multiple faults can be detected (with a belief value) and a list of the most probable faults is created. The shortfall of this approach is that the rules used at the D-S fusion step must be defined by the designer and require expert knowledge of the application under examination.

\section{HYBRID METHODS}

Data driven techniques have been used in combination to produce more accurate results or achieve a faster response. Fuzzy logic has been used in combination with ANN and SVM (section 5.1). PCA has been used in combination with ANN and wavelet transformation (section 5.2). In [103] there is developed a diagnostic algorithm by combining genetic algorithms with fuzzy logic applied at an HVAC system. Diagnostic rules, expressed by threshold values, for various system faults are generated by a fuzzy logic algorithm. An objective function having as variables the rules (thresholds) is created and a genetic algorithm is used to calculate the threshold values that optimize the objective function.

Algorithms created by combining more than one data driven technique remain a data driven approach. This section focuses on algorithms that combine model based and data driven techniques (hybrid methods). Hybrid methods aim to enhance the diagnostic results by leveraging the advantages and avoiding the limitations of their consisting techniques [104].

The authors in [105] apply a diagnostic methodology that combines the FDI method and the support vector machine technique on a vehicle's antilock braking system. A physical model of the system is created and four component faults and one sensor fault are considered into the analysis. The parity space technique (FDI), which has the advantage of faster response, is used to isolate the sensor faults and one component fault. The observer based (FDI) technique, which can construct residuals more robust to noise, is used to isolate the rest component faults. Finally, the SVM technique is used to isolate the two remaining component faults, which could not be distinguished using FDI approaches.

In [106] there is proposed a methodology that uses model based techniques for fault detection and data driven techniques for fault isolation. The proposed methodology is applied on a vehicle's electronic power system, observing six system values and considering four system component faults. The CUSUM algorithm (residual evaluation from the FDI technique) was used to detect faults in the system by comparing the cumulative sum of the squared residuals of the observed measurements to a predefined threshold. The fault isolation step consisted of three stages: dimensionality reduction, fault classification, severity estimation. Partial Least Square (PLS) algorithm was used to reduce the dimensionality of the dataset. The reduced dimensionality data was used to train machine learning algorithms (Support Vector Machine, Probabilistic Neural Network, and Nearest Neighbour) to classify different faults. Finally SVM regression is used to estimate the fault severity. The proposed methodology was tested on data collected by a simulation model. The results demonstrated high classification rate and fault severity estimation accuracy improved as the fault severity increased.

In [107] there is developed a hybrid fault diagnosis algorithm for fault detection and isolation. The proposed methodology was applied to an HVAC system. Simulations were conducted considering healthy conditions and three component faults under various severity degrees. At the fault detection stage, the moving average method (residual evaluation from the FDI technique) is used to detect a fault by evaluating the residual between healthy and faulty simulation 
cases. A SVM was trained to classify the faults under examination. This methodology was tested on simulation data and could accurately detect the injected faults.

\section{SYSTEM LEVEL DIAGNOSTICS}

An emerging topic among the diagnostic community during the last decade is the system level diagnostic approach. System level diagnostics targets in accurately assessing the system's health state and when a fault is detected, identifying the system's faulty component. The system's components considered in the analysis are defined by the designer and are the Line Replaceable Units (LRU) of each application. System level diagnostics is an approach that targets in informing the maintainer which LRU should be replaced when a system is not performing properly. A standard definition for system level diagnostics is still missing from the literature, but there are two major features that can be identified under this approach:

- Both structural and functional interconnection of the system components is being considered.

- When a faulty component has been identified, the diagnostic analysis is not continued in more detail to identify the root cause of the faulty component.

In [11] there is developed a system level diagnostic algorithm using a model based methodology applied on a helicopter's gearbox. Initially, a database is created that contains the system's building components, their possible fault modes and physical parameters (Condition Indicators - CI) that can capture these fault modes. Based on the system structure, a functional model that describes the functionality of each component is created. Each element of the functional model is correlated with one or more fault modes and each fault mode is associated to one or more condition indicators. Based on the relationship between the fault modes and the condition indicators, a faultsymptom matrix is created. Using this matrix, the faulty components can be uniquely identified. After the fault identification, using the system's structure, a fault propagation tree is created. This tree points out components that are probable of developing a fault, due to their adjacency to a faulty component. The proposed technique was applied on a helicopter's intermediate gearbox considering as condition indicators different frequency bands captured by two accelerometers at the input and at the output of the gearbox. The diagnostic analysis could isolate all component faults.

In [108] the authors apply system level diagnostic methodology on a Heat Ventilation and Air Condition (HVAC) system. The proposed algorithm consists of two modules, a sensor fault detection module and a component fault detection module. As regards the sensor fault detection module, Principal Component Analysis (PCA) was used to reduce the dimensionality of the data and the $\mathrm{Q}$ test was used to detect sensor faults. For the component diagnosis module, subsystems of the same type of components, e.g. a Heat Exchanger subsystem (consisting of all the Heat Exchangers of the system), were considered to break the system down to a subsystem level. Appropriate physical parameters for each subsystem were selected (Performance Indicators - PIs). The PIs reflect the system's health state and can detect faults in the corresponding subsystem group. This methodology could accurately detect sensor faults or high severity components faults when examined separately. Limitation of this methodology were that its accuracy in detecting faults was decreased in cases of low severity components faults and in cases were sensor and component faults were combined.

The authors in [109] developed a system level diagnostic method to detect HVAC component faults that lead to delta-T syndrome. Delta-T syndrome is a phenomenon where water temperature difference produced from an Air Handling Unit (AHU) is lower than the design value. In this application the subsystems considered are an Air Handling Unit and Heat Exchangers since these are the systems that can cause delta-T syndrome. For each one of them, appropriate physical variables (Performance Indices - PI), that can effectively capture each component's degradation, are selected. PI baselines are created offline based on healthy historic data. Residual between observed PI and baseline PI is compared to adaptive threshold that captures the variations due to operating conditions. When the residual exceeds a PI threshold a fault is isolated to the corresponding component. The proposed method was tested and could accurately detect and isolate faults into systems under examination when a low delta-T syndrome occurs.

In [110] there is developed a methodology of estimating the health state of a battery of an electric vehicle. Batteries for electric vehicles consist of many cells and the techniques that focus on the cell level diagnostics cannot guarantee accurate system level diagnostics. In this study, the battery cells can be connected either to the "main" output, which is used to supply the electric vehicle with power or to the "test" output, which is used for the diagnostic analysis. The diagnostic analysis conducted on the cells connected to the "test" output can use any component (cell) - level technique. Data collected from a simulation model under healthy and faulty conditions were used to conduct the diagnostic analysis. An experimental rig was used to validate the simulation model. A parameter estimation technique (from the FDI method) was used to assess each cell's health state (component level). It is concluded that as the number of cells connected to the test output increased, the accuracy of the diagnostic results increase as well. An important contribution of this methodology is that the cells 
used for diagnostics are randomly selected, thus the analysis is conducted at a system level.

In [111] there is developed a method of assessing the health state of a building's power consumption subsystems (HVAC, lighting, lift, etc.). The system's data were classified into working days and non-working days. Furthermore, depending on weather conditions and operating modes of the subsystems, data were separated into cooling or heating categories. A building's power consumption system was divided into four major subsystems and a diagnostic analysis based on different time periods (hourly, weekly, daily) was conducted. By combining physical parameters for every component, appropriate metrics (Energy Performance Indices - EPI) that describe its corresponding performance are created. Based on historic data, regression models create the baseline for each Energy Performance Index. Conducting a statistical analysis on the data, thresholds that can detect faults and suggest their severity can be defined. Weekly diagnosis could accurately detect the total building's power consumption faults. However, there were cases were various system faults oversubscribed with each other and the weekly diagnosis could not accurately isolate a specific fault. Daily and hourly diagnosis could identify subsystems that operated under faulty conditions. The specific component and the root cause of the failure could not be identified by the algorithm and physical inspection was required.

The authors in [112] applied a system level diagnostic methodology at an aircraft's Environmental Control System (ECS). The proposed method breaks the ECS down into two major subsystems and each subsystem into two major components and one sensor module. Initially, a sensor dependency matrix (D-Matrix) is created that identifies the sensors that can capture deviations in each component's health state. The fault detection strategy used traverses the system's pyramid top-down. Thus, at the first step, a subsystem that contains a fault is identified and at the second step, the specific faulty component or sensor module is isolated. The diagnostic analysis is based on neural networks. For training of each neural network for every subsystem and every component, there was considered a dataset for a healthy case and a dataset for a faulty case. The healthy case considered the subsystem or the component under examination to be healthy while one of the remaining components, in another subsystem, is faulty. The faulty case considered the component under examination to be faulty while all other components are healthy. The proposed methodology was tested and could accurately detect faults in all components under examination. Compared to an alternative training method which considered that at the healthy case every component was healthy and at the faulty case the component under examination being faulty and every other component healthy, the proposed training method demonstrated more accurate results. This proves that capturing the component's interconnections increases the diagnostic accuracy.

In [113] there is developed a methodology that can detect and isolate faults in an aircraft's fuel system, using a Bayesian network. The proposed method could accurately estimate the degree of degradation of the component under examination. The novelty introduced in this work is that the calculation of a component's degradation level considered other system components suffering from various degrees of degradation. A Bayesian network was constructed considering the probability of multiple components being degraded at the same time. The results demonstrated that following this technique the various components degradation levels could be accurately identified. Compared to a Bayesian network that did not consider the probability of various components being simultaneously degraded, the proposed methodology demonstrated higher accuracy.

In [114] there is developed a system level diagnostic approach applied on a HVAC system. The proposed method considers the system components interdependencies. At the first step, physical analysis of the system under examination is conducted and the most representative features that can accurately detect and isolate the components' failure modes are selected. The selected features are used to create a Bayesian network that connects features (symptoms) to the corresponding fault modes with their respective probabilities. The system faults under examination, as well as their respective a priori probabilities, are estimated using historic data. Optimal estimation techniques were used to capture variables necessary for the mass and energy balance in cases were physical sensors were missing. The proposed method was tested in two different case studies. In the first one a fault was considered only in one subsystem and in the second, faults could be present in two subsystems simultaneously. This study highlights that if the diagnostic analysis cannot capture the components interconnection in different hierarchical levels or the supplementary role various components have in the same level, the accuracy of the diagnostic results decreases. The case studies considered, demonstrated that when the components interconnections (mass and energy balance) are captured in the Bayesian network, the diagnostic results were more accurate.

\section{SUMMARY \& DISCUSSION}

This section consists of two parts, the first part summarizes and discusses diagnostic methods, highlighting advantages and shortfalls of each one. The second part analyses more thoroughly the system level diagnostic concept, aiming to emphasize capability for specific applications as well as this concept's strengths and weaknesses. Also, there is discussed the way existing diagnostic 
methods have been applied to system level diagnostics, areas for further research are proposed.

\subsection{Diagnostic Methods Summary}

Each of the diagnostic methods elaborated in the previous sections offers specific advantages and disadvantages and demand compliance with particular prerequisites for their successful implementation. Major considerations include:

i. Installed sensors or hardware;

ii. Online or offline application.

Discussion of advantages, disadvantages of each method as well as requirements for their successful application, was carried out at each respective section. This section offers a comprehensive summary of the pros and cons of each method and the availability of their application.

Model based methods define thresholds and rules for fault detection and isolation based on physics based simulation models. Analysis under this approach is highly dependent on modelling accuracy. This means that these methods are very useful for OEMs that have an accurate understanding of the system's behaviour (e.g. thermodynamic design analysis, structural design analysis). Accurate engineering understanding allows development of high fidelity models which can produce more precise diagnostics. System operators that are able to develop lower fidelity models require implementation of more complex fault diagnostic algorithms (e.g. adaptive thresholds, consecutive occurrence of faults) in order to achieve acceptable fault detection rates.

A design challenge faced by all diagnostic methods is false alarms to misdetections ratio. This problem usually arises due to noise or unmodelled parameters that exist in a system's operation. The advantage of model based methods is that this ratio can be controlled. A proposed technique controlling this ratio is by selecting appropriate fault detection thresholds. These thresholds can either be selected by the designer of the diagnostic algorithm (subjectively) or can be quantified and selected objectively [46]. The great advantage of model based analysis is that algorithms can be developed based on engineering knowledge of the system without the requirement of fault history of the system or component under examination. On the other hand, a disadvantage of this method is that complex systems cannot be easily modelled.

Data driven methods are based on machine learning and probability theory algorithms. Supervised or unsupervised learning algorithms are used to solve classification problems (classify systems or components healthy or faulty), probabilistic and stochastic algorithms are used to assign probabilities to systems or components associated with their health condition. Data driven algorithms are used in combination in many applications in order to combine high accuracy features (SVM, GA) with fast response features (neural networks). Results produced by data driven methods are based both on the quality of the data used for training the algorithm as well as the training strategy used. Good quality training datasets should contain a wide representation of the system and component failure modes with the corresponding physical parameter values. Lower quality datasets require more complex training strategies that may need to combine more than one algorithm. A disadvantage of the data driven methods is that they cannot be easily adjusted in order to optimize the rate of false alarms to misdetections and are referred to as "black box" methods. Data driven methods are useful in cases where complex systems are under examination and limited engineering understanding is available for their performance.

Section 3 illustrated that diagnostic methods based on expert systems require historic knowledge of the system faults as well as the corresponding maintenance actions. Even in cases where this information is available, it is challenging to interpret databases containing historic information and develop rules that can be used by computer algorithms. Also, the developed rules will be associated with systems working within a specific operational envelop and under common environmental conditions. However, diagnostic expert systems are valuable in cases where there are not sufficient sensors installed on the system under examination (legacy systems) and there is limited engineering knowledge for their performance.

Table 1 gives of tabulated representation of the pros and cons of each category of methods described above. Understanding the advantages, disadvantages and the necessary requirements for successful implementation for each one of them, allows the diagnostic engineer to select the most appropriate methods for the application under examination.

\subsection{Diagnostic Methods Summary}

System level diagnostics targets in accurately detecting faults in a system and identifying faulty LRUs. In industrial applications where unscheduled maintenance is expensive, prompt detection of faulty components that should be replaced is of great value.

A great strength of this approach is that it captures interconnections among system components. The analysis aims to identify fault symptoms that characterize each component's health state as well as cases where different components have identical fault symptoms.

Fundamental steps followed by system level diagnostics are:

- Identification of fault symptoms for all component

- Identification of cases in which a failure mode has unique fault symptoms 
Table 1 Advantages \& Shortfalls of Diagnostic Methods

\begin{tabular}{|c|c|c|}
\hline Method & Advantages & Shortfalls \\
\hline Model Based & $\begin{array}{l}\text { - Do not require historic knowledge of the } \\
\text { system in operation } \\
\text { - The rate of false alarms to misdetections can } \\
\text { be adjusted }\end{array}$ & $\begin{array}{l}\text { - Require knowledge of the engineering of } \\
\text { the system or component under } \\
\text { examination }\end{array}$ \\
\hline Data Driven & $\begin{array}{l}\text { - Can be used without knowledge of the } \\
\text { performance of the system or components } \\
\text { - Various algorithms can be easily combined } \\
\text { to enhance the results }\end{array}$ & $\begin{array}{l}\text { - The results depend on the quality of the } \\
\text { training datasets } \\
\text { - Algorithms are difficult to adjust for false } \\
\text { alarms to misdetection rate "black box } \\
\text { methods" }\end{array}$ \\
\hline $\begin{array}{l}\text { Rule \& Case Base } \\
\text { Reasoning }\end{array}$ & $\begin{array}{l}\text { A diagnostic analysis can be developed } \\
\text { without engineering knowledge of the system }\end{array}$ & $\begin{array}{l}\text { - Historic or expert knowledge of the system } \\
\text { is hard to acquire and transform in an } \\
\text { algorithm in many applications }\end{array}$ \\
\hline
\end{tabular}

- Identification of cases in which a component's fault symptom can be confused with other components fault symptoms

- Identification of ambiguity groups created

Table 2 summarizes reviewed publications of system level diagnostics presented in section 7, highlighting their main advantages and shortfalls. Most applications use a methodology of defining metrics or indicators that can be sensitive to a component's fault, but insensitive to all other components faults (physics based). Designing metrics or indicators that are sensitive to specific faults and insensitive to all other faults is similar to the idea of generating residuals that correspond to specific faults at FDI methods. The difference is that development of FDI residuals was based on intelligent matrix manipulation. In system level diagnostics, health metrics are based on engineering judgement and are selected by the designer. Defined metrics can be either individual physical parameters or a function of system's physical parameters. Health state of modelled components is associated with the defined metrics. These components may either represent an LRU or a group of LRUs.

Table 2 Applications of system level diagnostic approach

\begin{tabular}{|c|c|c|c|}
\hline Application & Method & Key Advantage & Key Shortfall \\
\hline Helicopter gearbox & $\begin{array}{l}\text { Physics based (Condition } \\
\text { Indicators) }\end{array}$ & $\begin{array}{l}\text { Monitoring one physical } \\
\text { parameter, all component } \\
\text { faults can be identified. }\end{array}$ & $\begin{array}{l}\text { Single physics system. } \\
\text { No sensor faults considered. }\end{array}$ \\
\hline $\begin{array}{l}\text { Heat Ventilation and } \\
\text { Air Condition }\end{array}$ & $\begin{array}{l}1^{\text {st }} \text { stage: PCA } \\
2^{\text {nd }} \text { stage: Physics based } \\
\text { (Performance Indicators) }\end{array}$ & $\begin{array}{l}\text { Both components and } \\
\text { sensor faults could be } \\
\text { identified. }\end{array}$ & $\begin{array}{l}\text { Single physics system. } \\
\text { When both sensor and } \\
\text { component faults were } \\
\text { considered, sensor fault } \\
\text { detection accuracy was } \\
\text { decreased. }\end{array}$ \\
\hline $\begin{array}{l}\text { Environmental Control } \\
\text { System }\end{array}$ & Artificial Neural Networks & $\begin{array}{l}\text { Faults could be detected on } \\
\text { major subsystems and } \\
\text { sensors. }\end{array}$ & $\begin{array}{l}\text { Single physics system. } \\
\text { Limited ECS subsystems were } \\
\text { considered. }\end{array}$ \\
\hline HVAC & $\begin{array}{l}\text { Physics based (Performance } \\
\text { Indicators) }\end{array}$ & $\begin{array}{l}\text { Accurate fault isolation on } \\
\text { the subsystems under } \\
\text { examination. }\end{array}$ & $\begin{array}{l}\text { Single physics system. } \\
\text { Limited HVAC subsystems } \\
\text { were considered. }\end{array}$ \\
\hline HVAC & Bayesian network & $\begin{array}{l}\text { Multiple faults could be } \\
\text { captured accurately. }\end{array}$ & $\begin{array}{l}\text { Single physics system. } \\
\text { No sensor faults considered. }\end{array}$ \\
\hline $\begin{array}{l}\text { Electrical vehicle } \\
\text { battery }\end{array}$ & $\begin{array}{l}\text { Accelerating aging test } \\
\text { (component level) } \\
\text { Separation of "main" output } \\
\text { cells and "test" output cells } \\
\text { (system level) }\end{array}$ & $\begin{array}{l}\text { All battery cells can be } \\
\text { tested. }\end{array}$ & $\begin{array}{l}\text { Single physics system. } \\
\text { Accuracy depends on the } \\
\text { number of the tested cells. }\end{array}$ \\
\hline Wind power plant & $\begin{array}{l}\text { Unsupervised learning } \\
\text { algorithms (DBSCAN, } \\
\text { spectral clustering, PCA) }\end{array}$ & $\begin{array}{l}\text { Fault detection capability } \\
\text { for the overall power plant. }\end{array}$ & $\begin{array}{l}\text { Single physics system. } \\
\text { The faulty components could } \\
\text { not be isolated. }\end{array}$ \\
\hline Building power system & Energy Performance Indices & $\begin{array}{l}\text { Fault on the major energy } \\
\text { consumption subsystems } \\
\text { were accurately identified. }\end{array}$ & $\begin{array}{l}\text { Single physics system. } \\
\text { The exact faulty components } \\
\text { could not be accurately } \\
\text { identified. }\end{array}$ \\
\hline Fuel system & Bayesian network & $\begin{array}{l}\text { Multicomponent } \\
\text { degradation. }\end{array}$ & $\begin{array}{l}\text { Single physics system. } \\
\text { No sensor fault considered. }\end{array}$ \\
\hline
\end{tabular}


Data driven approaches such as neural networks [112] and Bayesian networks [113] have also been used for system level diagnostics. The key element that allows data driven algorithms to capture components interconnections is the training strategy used. As an example, is presented the methodology used by [112], to train a neural network (The healthy case considered the subsystem or the component under examination to be healthy while one of the remaining components, in another subsystem, is faulty. The faulty case considered the component under examination to be faulty while all other components are healthy.)

However, there are still challenges in this area. System level diagnostic applications in the literature either consider a single sensor system [11] or a system operating under a single physical environment [108-110, 113]. Analysis of multiphysical systems has not been widely discussed.

Also, in cases where sensor and component faults were combined, diagnostic accuracy was lower compared to cases where each one of them was considered independently [108]. Methodologies more robust to combined sensor and component faults should be further developed.

An increasing trend among the diagnostic community is the integration of model based and data driven techniques (hybrid methods). In section 6 , publications of hybrid methods were presented. These hybrid algorithms produce more accurate results and require less computational time compared to model based or data driven methods individually. This area should be further developed and applied more widely to system level diagnostics.

Concluding, areas of further research that could enhance the performance of system level diagnostics and expand its applications are:

- Application of system level diagnostic approach on multiphysical systems

- Integration of sensor and component faults

- Further development of hybrid techniques and application on system level diagnostics

\section{CONCLUSIONS}

This work presents a review of system diagnostics. The necessity of this approach in optimizing maintenance tasks in high value assets is highlighted and examples of its applications have been given. The key features of this approach and techniques employed for their implementation have been presented. Strengths and limitations of this approach are presented and a discussion is conducted analysing the main reasons of the observed limitations and areas of further research were proposed.

Also, a literature review of the most recent diagnostic methodologies and diagnostic reasoning tools identified in the literature is conducted and a taxonomy is proposed. The wide spectrum of available diagnostic techniques is presented and their strengths and weaknesses are highlighted. This aims in informing the reader about the existing diagnostic methods and based on them introduce system level diagnostics. This is also important for the diagnostic engineer, since by understanding the main features, the advantages and disadvantages of diagnostic methods, the most appropriate technique(s) can be used to develop system level diagnostic algorithms on each application.

As a synopsis, the potential accuracy of the diagnostic results can be significantly improved in any given application by identifying the special features and by deploying the most compatible or a combination of most suitable diagnostic techniques. In addition, the proposed taxonomy can be regarded as an enabler to down select the most appropriate diagnostics technique, method, and any combination of methods best suited to diagnose a hardware system efficiently.

\section{SOURCE OF FUNDING}

The authors would like to thank The Boeing Company for funding this project.

\section{ACKNOWLEDGMENTS}

The authors would like to thank Cordelia Ezhilarasu, for her helpful insights and critical comments

\section{REFERENCES}

1. Jennions IK. editor. Integrated Vehicle Health Management - Perspectives on an Emerging Field. SAE International; 2011.

2. Mobley RK. An introduction to predictive maintenance. Elsevier; 2002.

3. Evan C P. Industrial Internet: Pushing the boundries of Minds and Machines 2012. Available from: http://www.ge.com/docs/chapters/Industrial_Internet. pdf

4. Esperon-Miguez M, John P, Jennions IK. A review of Integrated Vehicle Health Management tools for legacy platforms: Challenges and opportunities. Prog Aerosp Sci. 2013;56:19-34. http://dx.doi.org/10.1016/j.paerosci.2012.04.003

5. Pelham JG, Fan I-S, Jennions I, McFeat J. Application of an AIS to the problem of through life health management of remotely piloted aircraft. AIAA Infotech Aerosp. 2015;(January):5-9.

6. Piel Eric, Gonzalez-Sanchez A, Gross H, Gemund AJC Van. Online fault localization and health monitoring for software systems. in: situation awareness with systems of systems. New York: Springer Science+Business Media; 2013. 229-45. http://link.springer.com/10.1007/978-1-4614-6230-9

7. Ogaji SOT, Sampath S, Singh R, Probert SD. Parameter selection for diagnosing a gas-turbine's performance-deterioration. Appl Energy. 2002;73(1): $25-46$.

8. Staszewski WJ, Boller WJ, Tomlinson GR. Health monitoring of aerospace structures. 2004. 266. http://dx.doi.org/10.1002/0470092866 
9. Duffuaa SO, Ben-Daya M. Handbook of Maintenance Management and Engineering. Handbook of Maintenance Management and Engineering. 2009. 223-235:

http://www.scopus.com/inward/record.url?eid=2s2.0-84892338874\&partnerID=tZOtx3y1

10. Mehdi G, Naderi D, Ceschini G, Roshchin M. Modelbased reasoning approach for automated failure analysis: An Industrial Gas Turbine Application. 2011;1-10.

11. Saha B, Vachtsevanos G. A Model-based reasoning approach to system fault diagnosis. Proc 10th WSEAS Int Conf Syst. 2006;2006:64-71. http://dl.acm.org/citation.cfm?id=1984211.1984224

12. Bartlett LM, Hurdle EE, Kelly EM. Integrated system fault diagnostics utilising digraph and fault tree-based approaches. Reliab Eng Syst Saf. 2009;94(6):110715.

13. Papadopoulos Y. Model-based system monitoring and diagnosis of failures using statecharts and fault trees. Reliab Eng Syst Saf. 2003;81(3):325-41.

14. Singh S, Holland SW, Bandyopadhyay P. Trends in the development of system-level fault dependency matrices. IEEE Aerosp Conf Proc. 2010;

15. Durkin J. Expert systems: Design and development. New York: Macmillan Publishing Company; 1994.

16. Dubrova E. Fault-tolerant design. Fault-Tolerant Design. 2013. 1-185 p.

17. Ferrara LA. Summary description of the AAP Apollo telescope mount. 1968.

18. Shi N-Z, Tao J. Statistical hypothesis testing. Toh Tuck Link, Singapore: World Scientific Publishing Co. Pte. Ltd.; 2008.

19. Siegmund D. Sequential Analysis. New York: Springer; 1985.

20. Mohanty SR, Pradhan AK, Routray A. A cumulative sum-based fault detector for power system relaying application. IEEE Trans Power Deliv. 2008;23(1):7986.

21. Basseville M, Nikiforov I. Fault isolation for diagnosis: nuisance rejection and multiple hypotheses testing. Annu Rev Control. 2002;25(1):83-94. http://linkinghub.elsevier.com/retrieve/pii/S13675788 $\underline{01000050}$

22. Patton, R. J, Chen J. Review of parity space approaches to fault diagnosis for aerospace systems. J Guid Control Dyn. 1994;17(2).

23. Höfling T, Isermann R. Fault detection based on adaptive parity equations and single-parameter tracking. Control Eng Pract. 1996;4(10):1361-9.

24. Patton RJ, Chen J. Robust fault detection using eigenstructure assignement: a tutortial considerations and some new results. In: Proceedings of the 30th conference on decision and control. Brighton, England; 1991.

25. White J, Speyer J. Detection filter design: Spectral theory and algorithms. IEEE Trans Automat Contr 1987;32(7):593-603.

http://ieeexplore.ieee.org/document/1104682/

26. Frank PM. Analytical and Qualitative Model-based Fault Diagnosis - A Survey and Some New Results. Vol. 2, European Journal of Control. 1996. p. 6-28.

27. Hwang I, Kim S, Kim Y, Seah CE. A survey of fault detection, isolation, and reconfiguration methods. IEEE Trans Control Syst Technol. 2010;18(3):63653.
28. Patton RJ, Frank PM, Clarke RN. Fault diagnosis in dynamic systems: theory and application. Upper Saddle River, NJ, USA: Prentice-Hall, Inc.; 1989.

29. Frank PM. Fault diagnosis in dynamic systems using analytical knowledge-based redundancy - A survey and some new results. Automatica. 1990;26(3):45974 .

30. Isermann R. Fault diagnosis of machines via parameter estimation and knowledge processingTutorial paper. Automatica. 1993;29(4):815-35.

31. Isermann R. Process Fault Detection Based on Modeling and Estimation Methods-A Survey. 1984;4(4).

32. Volponi AJ. Foundations of Gas Path Analysis I. In: Gas turbine condition monitoring and fault diagnosis - Lecture series 2003-01. von Karman Institute for Fluid Dynamics; 2003.

33. Volponi AJ. Foundation of Gas Path Analysis II. In: Gas turbine condition monitoring and fault diagnosis - Lecture series 2003-01. von Karman Institute for Fluid Dynamics;

34. Doel DL. An Assessment of Weighted-Least-Squares Based Gas Path. In: International gas turbinbe and aeroengine congress and exposition. Cincinati, Ohio: ASME; 1993.

35. Moseler O, Isermann R. Application of model-based fault detection to a brushless DC motor. IEEE Trans Ind Electron. 2000;47(5):1015-20.

36. Urban LA. Gas path analysis applied to turbine engine condition monitoring. $\mathrm{J}$ Aircr. 1973;10(7):400-6.

37. Doel DL. Development of baselines, influence coefficients and statistical inputs for gas path analysis. In: Gas turbine condition monitoring and fault diagnosis - Lecture series 2003-01. von Karman Institute for Fluid Dynamics; 2003

38. Stamatis AG. Optimum Use of Existing Sensor Information for Gas Turbine Diagnostics. In: Proceedings of the ASME Turbo Expo 2008 http://proceedings.asmedigitalcollection.asme.org/pro ceeding.aspx ?articleid $=1623480$

39. Volponi AJ. Gas Turbine Engine Health Management: Past, Present, and Future Trends. J Eng Gas Turbines Power. 2014;136(5):051201. http://gasturbinespower.asmedigitalcollection.asme.or g/article.aspx?doi=10.1115/1.4026126

40. A.Stamatis, K. Mathioudakis, G. Berrios KP. Jet engine fault detection with discrete operating points gas path analysis. J Propuls Power. 1991;7(6).

41. Doel DL. TEMPER-A Gas-Path Analysis Tool for Commercial Jet Engines. J Eng Gas Turbines Power 1994;116(1):82. http://link.aip.org/link/JETPEZ/v116/i1/p82/s1\&Agg $=$ doi

42. Davis R. Diagnostic reasoning based on structure and behavior. Artif Intell. 1984;24(1-3):347-410. http://linkinghub.elsevier.com/retrieve/pii/000437028 4900420

43. Kolcio K, Hanson M, Fesq L. Validation of Autonomous Fault Diagnostic Software. IEEE. 1998;

44. Fesq L. Marple: An autonomous diagnostician for isolationg system hardware failures. UCLA; 1993.

45. Kolcio K, Breger L, Zetocha P. Model-based fault management for spacecraft autonomy. IEEE Aerosp Conf Proc. 2014;

46. Rossi C. Vehicle Health Monitoring Using Stochastic Constraint Suspension. In: AIAA Guidance, 
Nagivation, and Control Conference. Minneapolis, Minnesota; 2012.

47. Rossi C, Benson D, Sargent R, Breger L. ModelBased Design for Vehicle Health Monitoring. Infotech@aerosp 2012. 2012;(June):1-23. http://arc.aiaa.org/doi/abs/10.2514/6.2012-2577

48. Kolcio K, Fesq L. Model-based off-nominal state isolation and detection system for autonomous fault management. IEEE Aerosp Conf Proc. 2016;2016June.

49. De Kleer J, Kurien J. Fundamentals of model-based diagnosis. IFAC Proc. 2003;6670(June 2003):25. http://books.google.com/books?hl=en\&amp;lr=\&amp ;id=QAU9N34xOSsC\&amp;oi=fnd\&amp;pg=PA25 \&amp;dq=FUNDAMENTALS+OF+MODELBASED+DIAGNOSIS\&amp;ots=u6WQ2IY3yc\&am p;sig=MXiYjJExLVQFazdvBGIrdBgfzng

50. Stern R, Kalech M, Rogov S, Feldman A. How many diagnoses do we need? Artif Intell. 2017;248:26-45. http://dx.doi.org/10.1016/j.artint.2017.03.002

51. Feldman A, Provan G, Van Gemund A. Approximate model-based diagnosis using greedy stochastic search. J Artif Intell Res. 2010;38:371-413.

52. Keren B, Kalech M, Rokach L. Model-based diagnosis with multi-label classification. 22nd Int Work Princ Diagnosis, DX. 2011;

53. Kolcio K, Fesq L, Mackey R. Model-based approach to rover health assessment for increased productivity. 2017;

54. Mosterman PJ, Kapadia R, Biswas G. Using bond graphs for diagnosis of dynamic physical systems. 1995.

55. Daigle M, Field M, Foygel M. Model-based Diagnostics for Propellant Loading Systems. 2011;

56. Mosterman PJ, Biswas G. Diagnosis of continuous valued systems in transient operating regions. IEEE Trans Syst Man, Cybern Part ASystems Humans. 1999;29(6):554-65.

57. Narasimhan S, Biswas G. Model-Based Diagnosis of Hybrid Systems. Syst Man Cybern Part A Syst Humans, IEEE Trans. 2007;37(3):348-61. http://ieeexplore.ieee.org/stamp/stamp.jsp?tp=\&arnu mber $=4154930$

58. Chen B, Li J, Wei G, Ma B. A novel localized and second order feature coding network for image recognition. Pattern Recognit. 2018;76:339-48.

59. Wang S, Li G, Bao Y. A novel improved fuzzy support vector machine based stock price trend forecast model. 2018. http://arxiv.org/abs/1801.00681

60. Vapnik V. The nature of statistical learning theory. Springer; 1995. http://www.ncbi.nlm.nih.gov/pubmed/18255760

61. Simon Haykin. Neural networks: a comprehensive foundation. The Knowledge Engineering Review. 1999; 13:409-12.

62. Winston PH. Artificial Intelligence. 3rd ed. AddisonWesley; 1993.

63. Nocedal J, Wright S. Numerical Optimization. New York: Springer; 1999.

64. Muhammad MB, Sarwar U, Tahan MR, Karim ZAA. Fault diagnostic model for rotating machinery based on principal component analysis and neural network. ARPN J Eng Appl Sci. 2016;11(24):14327-31.

65. Kanelopoulos K, Stamatis A, Mathioudakis K. Incorporating Neural networks into gas turbine performance diagnostics. ASME 1997 Int Gas Turbine Aeroengine Congr Exhib. 1997; V004T15A011-V004T15A011.
66. Sina Tayarani-Bathaie S, Sadough Vanini ZN, Khorasani K. Dynamic neural network-based fault diagnosis of gas turbine engines. Neurocomputing 2014;125:153-65. http://dx.doi.org/10.1016/j.neucom.2012.06.050

67. Pascoal RM, Vianna WOL, Gomes JPP, Galvão RKH. Estimation of APU failure parameters employing linear regression and neural networks. Phm. 2013;1-7.

68. Volponi AJ, DePold H, Ganguli R, Daguang C. The use of Kalman filter and neural network methodologies in gas turbine performance diagnostics: A Comparative Study. J Eng Gas Turbines Power. 2003;125(4):917-24. http://dx.doi.org/10.1115/1.1419016

69. Zedda M, Singh R. Fault diagnosis of a turbofan engine using neural networks - A quantitative approach. 34th AIAA/ASME/SAE/ASEE Jt Propuls Conf Exhib. 1998;2.

http://arc.aiaa.org/doi/10.2514/6.1998-3602

70. Kramer MA. Autoassociative neural networks. Comput Chem Eng. 1992;16(4):313-28.

71. Uluyola O, Buczaka A, Nwadiogbub E. Neural networks based sensor validation and recovery methodology for advanced aircraft engines. In: Component and System Diagnostics, Prognosis, and Health Management. 2001: 102-9.

72. Guo T-H, Saus J, Lin C-F, Ge J-H. Sensor validation for turbofan engines using an autoassociative neural network. Guid Navig Control Conf. 1996; http://arc.aiaa.org/doi/10.2514/6.1996-3926

73. Zedda M, Singh R. Neural-network-based sensor validation for gas turbine test bed analysis. 2001;215:47-56.

74. Sadough Vanini ZN, Meskin N, Khorasani K. Multiple-Model Sensor and Components Fault Diagnosis in Gas Turbine Engines Using Autoassociative Neural Networks. J Eng Gas Turbines Power. 2014;136(9):091603.

http://gasturbinespower.asmedigitalcollection.asme.or g/article.aspx?doi=10.1115/1.4027215

75. Vapnik V. Support Vector Networks. Mach Learn 1995;20(3):273-297. http://link.springer.com/10.1007/BF00994018

76. Zhao Q, Wang B, Zhou G, Zhang W, Guan X, Feng $\mathrm{W}$, et al. An Improved Fault Diagnosis Approach Based on Support Vector Machine. In: 2016 IEEE International Conference on Prognostics and Health Management (ICPHM). 2016.

77. Vieira FM, Bizarria CDO. Health monitoring using support vector classification on an auxiliary power unit. In: IEEE Aerospace Conference Proceedings. 2009.

78. Zhou D, Zhang H, Weng S. A New Gas Path Fault Diagnostic Method of Gas Turbine Based on Support Vector Machine. J Eng Gas Turbines Power 2015;137(10):6

http://gasturbinespower.asmedigitalcollection.asme.or g/article. aspx? articleid $=2238484 \% 7 \mathrm{~B} \& \% 7$ DresultCli $\underline{\mathrm{ck}=3}$

79. Hou C, Wang Q. Diagnosis of Aircraft Engine Performance Deterioration Based on Support Vector Machines. 2014;1-5.

80. Nieto Gonzalez JP. Vehicle fault detection and diagnosis combining an AANN and multiclass SVM. Int J Interact Des Manuf. 2017;1-7. 
81. Frank PM, Köppen-Seliger B. Fuzzy logic and neural network applications to fault diagnosis. Int $\mathbf{J}$ Approx Reason. 1997;16(1):67-88.

82. Ross TJ. Fuzzy logic with engineering applications. West Sussex, England: John Wiley \& Sons, Ltd; 2004.

83. Allen WH, Rubaai A, Chawla R. Fuzzy neural network-based health monitoring for HVAC system variable-air-volume unit. IEEE Trans Ind Appl. 2016;52(3):2513-24.

84. Hang J, Zhang J, Cheng M. Application of multi-class fuzzy support vector machine classifier for fault diagnosis of wind turbine. Fuzzy Sets Syst. 2016;297:128-40.

http://dx.doi.org/10.1016/j.fss.2015.07.005

85. Wang Z, Zhao N, Wang W, Tang R, Li S. A Fault diagnosis approach for gas turbine exhaust gas temperature based on fuzzy c-means clustering and support vector machine. Math Probl Eng. 2015;2015:1-11.

http://www.hindawi.com/journals/mpe/2015/240267/

86. S.N.Sivanandam, S.N.Deepa. Introduction to genetic algorithms. Springer; 2008.

87. Zedda M, Singh R. Gas turbine engine and sensor fault diagnosis using optimization techniques. J. Propuls Power. 2002;18(5):1019-25. http://www.scopus.com/inward/record.url?eid=2s2.0-0036748352\&partnerID=tZOtx3y1

88. Kobayashi T, Simon DL. A hybrid neural network genetic algorithm technique for aircraft engine performance diagnostics. J Propuls Power. 2005;21(4):751-8.

89. Sampath S, Singh R. An integrated fault diagnostics model using genetic algorithm and neural networks. $\mathbf{J}$ Eng Gas Turbines Power. 2006; 128:49.

90. Ogaji SOT, Sampath S, Marinai L, Singh R, Probert SD. Evolution strategy for gas-turbine faultdiagnoses. Appl Energy. 2005;81(2):222-30.

91. Kong C, Kang M, Park G. Study on condition monitoring of 2-spool turbofan engine using nonlinear gas path analysis method and genetic algorithms. Int J Mater Mech Manuf. 2013;1(2):21420.

http://www.ijmmm.org/index.php?m=content\&c=ind ex\&a=show\&catid $=23 \& i d=55$

92. Johnson RA, Wichern DW. Applied Multivariate Statistical Analysis. Pearson Education International. 2008. 1-773.

https://www.pearsonhighered.com/program/JohnsonApplied-Multivariate-Statistical-Analysis-6thEdition/PGM274834.html\%5Cnhttp://pubs.amstat.or g/doi/abs/10.1198/tech.2005.s319

93. Zhou J, Guo A, Celler B, Su S. Fault detection and identification spanning multiple processes by integrating PCA with neural network. Appl Soft Comput J. 2014;14:4-11.

http://dx.doi.org/10.1016/j.asoc.2013.09.024

94. Misra M, Yue HH, Qin SJ, Ling C. Multivariate process monitoring and fault diagnosis by multi scale PCA. Comput Chem Eng. 2002;26:1281-93.

95. Li S, Wen J. A model-based fault detection and diagnostic methodology based on PCA method and wavelet transform. Energy Build 2014;68:63-71. http://dx.doi.org/10.1016/j.enbuild.2013.08.044

96. Jensen $\mathrm{F}$ V. Introduction to Bayesian networks. Springer; 2000.

97. Sedighi T, Phillips P, Foote PD. Model-based intermittent fault detection. Procedia CIRP [Internet].
2013;11:68-73.

http://dx.doi.org/10.1016/j.procir.2013.07.065

98. Jun H-B, Kim D. A Bayesian network-based approach for fault analysis. Expert Syst Appl 2017; 81:332-48.

http://linkinghub.elsevier.com/retrieve/pii/S09574174 17302191

99. Zhao Y, Wen J, Xiao F, Yang X, Wang S. Diagnostic Bayesian networks for diagnosing air handling units faults - part I: Faults in dampers, fans, filters and sensors. Appl Therm Eng. 2017;111:1272-86. http://dx.doi.org/10.1016/j.applthermaleng.2015.09.1 21

100. Zhao Y, Wen J, Wang S. Diagnostic Bayesian networks for diagnosing air handling units faults Part II: Faults in coils and sensors. Appl Therm Eng 2015;90:145-57. http://dx.doi.org/10.1016/j.applthermaleng.2015.07.0 $\underline{01}$

101. Shafer G. A Mathematical theory of evidence. Princeton University Press; 1976.

102. Guralnik V, Mylaraswamy D, Voges H. On handling dependent evidence and multiple faults in knowledge fusion for engine health management. Knowl Creat Diffus Util. 2002;

103. Lo CH, Chan PT, Wong YK, Rad AB, Cheung KL. Fuzzy-genetic algorithm for automatic fault detection in HVAC systems. Appl Soft Comput J. 2007; 7(2):554-60.

104. Eker ÖF. A Hybrid Prognostic Methodology and its Application to Well- Controlled Engineering. Vol. 53, Cranfield University. 2013.

105. Luo J, Namburu M, Pattipati KR, Qiao L, Chigusa $\mathrm{S}$. Integrated model-based and data-driven diagnosis of automotive antilock braking systems. IEEE Trans Syst Man, Cybern Part ASystems Humans. 2010; 40(2):321-36.

106. Ghimire R, Sankavaram C, Ghahari A, Pattipati K, Ghoneim Y, Howell M, et al. Integrated model-based and data-driven fault detection and diagnosis approach for an automotive electric power steering system. AUTOTESTCON (Proceedings). 2011; 1001445:70-7.

107. Liang J, Du R. Model-based fault detection and diagnosis of HVAC systems using support vector machine method. Int J Refrig. 2007;30(6):1104-14.

108. Wang S, Zhou Q, Xiao F. A system-level fault detection and diagnosis strategy for HVAC systems involving sensor faults. Energy Build. 2010;42(4): 477-90. http://dx.doi.org/10.1016/j.enbuild.2009.10.017

109. Gao D ce, Wang S, Shan K, Yan C. A system-level fault detection and diagnosis method for low delta-T syndrome in the complex HVAC systems. Appl Energy 2016;164:1028-38. http://dx.doi.org/10.1016/j.apenergy.2015.02.025

110. Ozkurt C, Camci F, Atamuradov V, Odorry C. Integration of sampling based battery state of health estimation method in electric vehicles. Appl Energy 2016;175:356-67. http://dx.doi.org/10.1016/j.apenergy.2016.05.037

111. Wang H, Xu P, Lu X, Yuan D. Methodology of comprehensive building energy performance diagnosis for large commercial buildings at multiple levels. Appl. Energy. 2016;169:14-27. http://dx.doi.org/10.1016/j.apenergy.2016.01.054 
112. Hare J, Gupta S, Najjar N, Orlando PD, Walthall R. System-level fault diagnosis with application to the environmental control system of an aircraft. 2015;

113. Lin Y, Zakwan S, Jennions I. A Bayesian approach to fault identification in the presence of multicomponent degradation. Int $\mathrm{J}$ Progn Heal Manag. $2017 ; 8$.

114. Verbert K, Babuška R, De Schutter B. Combining knowledge and historical data for system-level fault diagnosis of HVAC systems. Eng Appl Artif Intell. 2017;59:260-73.

Received 2018-08-29

Accepted 2018-11-15

Available online 2018-11-22

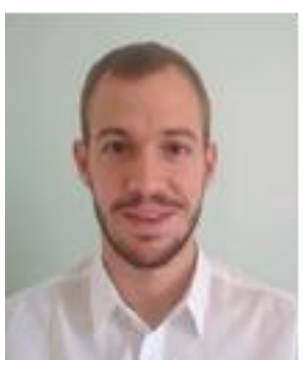

Christos SKLIROS holds a B.Eng. in Aeronautical Engineering from Hellenic Air Force Academy (2013) and a M.Sc. in Applied Mathematics from National Technical University of Athens (2016). $\mathrm{He}$ is currently a Ph.D. candidate at Cranfield University in the field of System Diagnostics and Health Management emphasizing in aerospace systems and Auxiliary Power Units (APUs). His research interests include also UAV technology and applications. He has worked as a maintenance engineer for Hellenic Air Force.

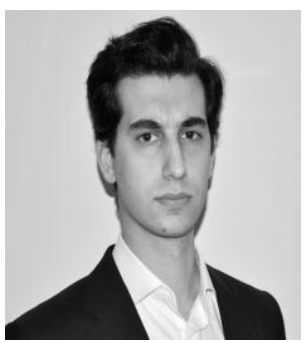

Dr. Manuel ESPERON specialises on design of IVHM systems for new and legacy platforms. He has been involved in projects to develop new design methodologies for IVHM, condition monitoring for thermal and electromechanical systems, the development of new data driven techniques, combining IVHM with Additive Manufacturing, financial analysis of IVHM solutions, and certification of IVHM systems. In the past he also conducted research on Energy Storage Systems. Dr Esperon has a Master's degree in Mechanical Engineering from the Technical University of Madrid, an $\mathrm{MSc}$ in Aerospace Engineering from Brunel University, and a PhD in IVHM from Cranfield University.

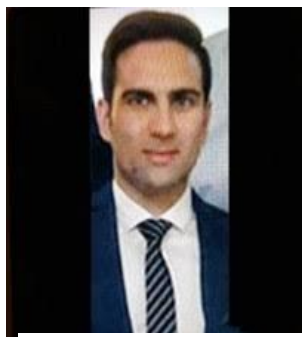

Dr. Ali FAKHRE is a research fellow in simulation \& test at the Cranfield University, IVHM Centre. He holds a Mechanical engineering degree and an engineering doctorate in Aerospace Propulsion both from Cranfield University, UK. In his current role he is responsible for the successful planning, implementation and delivery of TRL $4-5$ level projects, dedicated towards identifying new IVHM solutions for various major civil aircraft subsystems i.e. APU, ECS. He is a chartered Mechanical Engineer and a member of IMechE and RAeS.

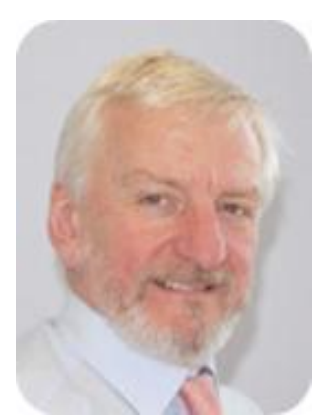

Prof. Ian JENNIONS's career spans some 40 years, working mostly for a variety of gas turbine companies. He has a Mechanical Engineering degree and a $\mathrm{PhD}$ in $\mathrm{CFD}$ both from Imperial College, London. He has worked for Rolls-Royce (twice), General Electric and Alstom in a number of technical roles, gaining experience in aerodynamics, heat transfer, fluid systems, mechanical design, combustion, services and IVHM. He moved to Cranfield in July 2008 as Professor and Director of the newly formed IVHM Centre. The Centre is funded by a number of industrial companies, including Boeing, BAE Systems, Thales, Meggitt, MOD, DRS, Alstom Transport and Novartis. He has led the development and growth of the Centre, in research and education, since its inception.

Ian is on the editorial Board for the International Journal of Condition Monitoring, a Director of the PHM Society, chair of the SAE IVHM Steering Group and contributing member of the HM-1 IVHM committee, a Chartered Engineer and a Fellow of IMechE, RAeS and ASME. He is the editor of five recent SAE books on IVHM and a co-author of the book: 'No Fault Found The Search for the Root Cause'. 